\title{
Linx
}

Revue des linguistes de l'université Paris X Nanterre

$55 \mid 2006$

Thème et thématisation

\section{La quantification adverbiale et l'indéfini objet des phrases génériques}

\section{Svetlana Vogeleer}

\section{OpenEdition}

\section{Journals}

Édition électronique

URL : http://journals.openedition.org/linx/468

DOI : $10.4000 /$ linx.468

ISSN : 2118-9692

Éditeur

Presses universitaires de Paris Nanterre

\section{Édition imprimée}

Date de publication : 1 décembre 2006

Pagination : 153-172

ISSN : 0246-8743

\section{Référence électronique}

Svetlana Vogeleer, «La quantification adverbiale et l'indéfini objet des phrases génériques », Linx [En ligne], 55 | 2006, mis en ligne le 23 février 2011, consulté le 19 avril 2019. URL : http:// journals.openedition.org/linx/468; DOI : 10.4000/linx.468 


\title{
La quantification adverbiale et l'indéfini objet des phrases génériques*
}

\author{
Svetlana Vogeleer \\ Institut Libre Marie Haps \& LTPC (Université Libre de Bruxelles) \\ vogeleer@skynet.be
}

\section{Introduction}

Cette étude examine les phrases génériques avec les adverbes quantificationnels souvent et toujours en position postverbale et un syntagme nominal ( $\mathrm{SN}$ ) indéfini en position de sujet et d'objet. C'est le SN objet qui sera au centre de notre discussion. Sur le plan de la structuration de l'information, l'analyse porte essentiellement sur les phrases avec une structure catégorique de base $\left[\mathrm{Un} \mathrm{N}_{\mathrm{suj}}\right]_{\mathrm{T}}\left[\mathrm{V}_{\mathrm{tr}} \mathrm{Q}-\mathrm{Adv} \text { un } \mathrm{N}_{\mathrm{obj}}(\mathrm{X})\right]_{\mathrm{F}}$, où $\mathrm{T}$ est un topique (ou thème), F est un focus (ou rhème, commentaire, propos) et Qadv est un adverbe quantificationnel (nous reviendrons sur cette terminologie dans les sections 2 et 3). Notre objectif est de définir les restrictions que cette configuration impose sur l'indéfini objet.

Cette problématique nous est suggérée par l'observation suivante. On considère souvent qu'un Q-adverbe est apte à multiplier un indéfini singulier (cf. par exemple de Swart, 2006). On constate cependant que cette aptitude des Q-adverbes est soumise à des restrictions en ce qui concerne l'indéfini singulier en position d'objet dans les phrases génériques. Certaines phrases avec un indéfini objet en position finale ne suscitent pas de discussions quant à leur acceptabilité (ex. 1), certaines autres sont

\footnotetext{
* Je remercie Marc Dominicy et le relecteur anonyme pour leur lecture attentive d'une version précédente de ce texte. Leurs critiques stimulantes et leurs suggestions généreuses m'ont permit de jeter un regard nouveau sur plusieurs points discutés.
} 
jugées comme «bizarres », " peu naturelles» (ex. 2). Le problème d'acceptabilité ne se pose pas lorsque l'indéfini est suivi d'un modifieur, nominal (un adjectif, une relative) ou verbal (un adverbe, un gérondif), ou d'un syntagme prépositionnel (SP) (ex. 3). Notre objectif est donc de définir les conditions qui autorisent ou bloquent l'indéfini singulier en position d'objet.

(1) a. [Un adjectif $]_{\mathrm{T}}$ [suit souvent un nom $]_{\mathrm{F}}$.

b. [Un cowboy $]_{\mathrm{T}}[\text { porte toujours un revolver }]_{\mathrm{F}}$.

c. [Un Cubain $]_{\mathrm{T}}[\text { fume souvent un cigare }]_{\mathrm{F}}$.

(2) a. ?? [n linguiste $]_{\mathrm{T}}[\text { publie souvent un article }]_{\mathrm{F}}$.

b. ??[Un singe $]_{\mathrm{T}}$ [mange souvent une banane $]_{\mathrm{F}}$.

c. ?? Un castor $]_{\mathrm{T}}[\text { construit toujours un barrage }]_{\mathrm{F}}$.

(3) a. Un linguiste publie souvent un article que personne ne lit.

b. Un singe mange souvent une banane en l'épluchant soigneusement.

c. Un castor construit toujours un barrage qui résiste à toutes les intempéries.

d. Un castor construit souvent un barrage à la lisière du bois.

La structure de l'article se présente comme suit. Dans la section 2, nous passerons brièvement en revue les particularités des Q-adverbes. La section 3 présente la structure catégorique. Dans la section 4, nous discuterons les particularités de la quantification adverbiale en tant que quantification sur les situations. Dans la section 5, l'analyse porte sur le cas où le SN objet indéfini est suivi d'un modifieur ou d'un SP. Dans les sections 6 et 7, nous proposerons notre analyse du SN objet indéfini en position finale.

\section{Q-adverbes}

Les adverbes quantificationnels quantifient sur les temps ou sur les événements (Abeillé et al., 2004). Ce dernier terme sera remplacé plus bas par celui de situation (cf. section 4). Dans notre analyse de la quantification adverbiale, nous utiliserons le terme de $Q$-adverbe de manière très restrictive en l'appliquant tout d'abord à un seul type d'adverbes quantificationnels: les adverbes de fréquence souvent, toujours, jamais, rarement, parfois/quelquefois, de temps en temps, tout le temps ${ }^{1}$, parmi lesquels nous opérerons une sélection supplémentaire qui ne nous laissera que les adverbes souvent et toujours.

Les adverbes de fréquence énumérés ci-dessus s'opposent aux adverbes d'habitude (babituellement, généralement, d'ordinaire), que Molinier \& Levrier (2000, p.79) classent non pas parmi les adverbes de fréquence, mais parmi les adverbes d'attitude. Comme tous les adverbes d'attitude, les adverbes d'habitude sont des adverbes phrastiques quelle que soit leur position dans la phrase. Quant aux adverbes de fréquence, on considère

\footnotetext{
1 Nous ne tenons pas compte des adverbes de fréquence en -ment (fréquemment, occasionnellement), qui, étant dérivés des adjectifs, ont des propriétés qui les rapprochent des adverbes de manière. L'adverbe rarement est mentionné dans notre série parce qu'il représente la contrepartie négative de souvent.
} 
généralement qu'ils peuvent avoir une lecture phrastique ou une lecture intégrée lorsqu'ils se trouvent en position postverbale (cf. Bonami et al., 2004). Notre analyse ne tiendra compte que des cas où le Q-adverbe se trouve dans cette position « ambiguë ».

Les adverbes d'habitude se distinguent des adverbes de fréquence par plusieurs propriétés distributionnelles ${ }^{2}$, parmi lesquelles Molinier \& Levrier (2000, p. 83-85) mentionnent notamment $\mathrm{l}^{\prime}(\mathrm{im})$ possibilité de focalisation au moyen d'une clivée : ${ }^{*} C^{\prime} e s t$ habituellement que Max déjeune à la cantine vs. C'est souvent que Max déjeune à la cantine. Nous ne nous occuperons pas ici des adverbes d'habitude.

En ce qui concerne les adverbes de fréquence, de Swart \& Molendijk (1998) distinguent entre les adverbes indépendants et les adverbes dépendants. Selon de Swart \& Molendijk (1998), les adverbes de fréquence dépendants (de temps en temps, tout le temps) diffèrent des adverbes indépendants en ce qu'ils sont toujours interprétés par rapport à une situation spécifique introduite dans le contexte précédent. Ainsi en (4a), l'adverbe de fréquence indépendant toujours autorise-t-il une suite comme (iii). En (4b), où l'adverbe de fréquence dépend de la situation introduite en (i), la suite (iii) pose un problème de cohérence.

(4) a.(i) Pauline jouait dans le jardin. (ii) Jean la harcelait toujours. (iii) Mais ce jour-là, il ne la harcelait pas.

b.(i) Pauline jouait dans le jardin. (ii) Jean la harcelait tout le temps.

(iii) ??Mais ce jour-là, il ne la harcelait pas.

(de Swart \& Molendijk, 1998, ex. 14-15)

Les adverbes de fréquence indépendants penvent avoir une lecture dépendante, tandis que les adverbes dépendants imposent cette lecture. C'est la raison pour laquelle ils seront exclus de notre analyse.

Parmi les adverbes qui nous restent, jamais et rarement sont des Q-adverbes négatifs. Si la valeur négative de jamais est évidente, celle de rarement nécessite une justification. La classification de rarement parmi les quantificateurs négatifs est due à sa monotonie décroissante. Avec rarement, les inférences monotones décroissantes peuvent aller jusqu'à zéro, ce qui explique l'incohérence de oui dans le dialogue suivant :

(5) - Pierre lit-il des livres ?

- *Oui, Pierre lit rarement des livres.

Étant lui-même négatif, rarement peut difficilement se trouver dans la portée de la négation (sauf s'il y a une reprise échoïque) :

(6) *Pierre ne lit pas rarement des livres.

Outre sa valeur négative, rarement se distingue de sa contrepartie positive sowvent en ce qu'il est dérivé d'un adjectif (rare $>$ rarement), ce qui le rapproche des adjectifs de manière (cf. note 1). Une autre différence capitale entre ces deux adverbes réside dans leur portée vis-à-vis d'un indéfini. En (7a), qui est une phrase bizarre dans la lecture

2 Je remercie le relecteur anonyme d'avoir attiré mon attention sur ce fait. Beaucoup d'autres observations concernant les Q-adverbes sont dues à ses commentaires judicieux et pertinents. 
par défaut, souvent a une portée large sur l'indéfini objet. En (7b), dont l'acceptabilité ne suscite pas d'objections, c'est l'indéfini qui a une portée large par rapport à rarement. Cette absence de portée sur un indéfini singulier caractérise également le quantificateur négatif jamais ${ }^{3}$.

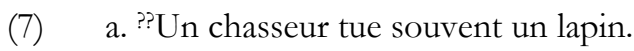

b. Un chasseur tue rarement un lapin.

c. Un chasseur ne tue jamais un lapin.

Comme jamais et rarement n'impliquent pas l'existence d'un référent dans les situations sur lesquelles ils quantifient, par exemple l'existence d'un lapin en (7), nous les écartons également.

Parmi les survivants, seuls souvent et toujours sont des quantificateurs forts, c'està-dire, dans les termes de la théorie des quantificateurs généralisés (Barwise \& Cooper, 1981), des quantificateurs qui établissent une relation entre deux ensembles. A la différence de souvent et toujours, parfois et quelquefois sont des quantificateurs faibles (intersectifs). Tout comme leur contrepartie nominale quelques, ils effectuent une quantification existentielle sans établir une relation avec un ensemble de référence ${ }^{4}$. A la différence de souvent et toujours, ils ne prennent pas de portée étroite par rapport à la négation (ex. 8). C'est pourquoi ils se placent normalement avant la négation postverbale. En (8a), les adverbes quantifient sur des situations dans lesquelles un chat mange une banane. En (8b), parfois quantifie sur des situations dans lesquelles un chat ne mange PAS une banane. Cela prouve que parfois/quelquefois se comportent toujours comme des adverbes phrastiques, c'est-à-dire comme en (8c).

(8) a. Un chat ne mange pas souvent/pas toujours une banane.

b. Un chat ne mange * pas parfois/ne mange parfois pas une banane.

c. Parfois, un chat ne mange pas une banane.

Doetjes (2002) analyse l'élément -fois de parfois et quelquefois comme un classifieur qui rend ces adverbes aptes à compter des événements à la manière des expressions numériques de type deux fois (à propos de ces expressions, cf. Landman, 2006). Selon Doetjes (2002), l'adverbe souvent contient ce même classifieur sous une forme implicite. Cependant, les propriétés de parfois et quelquefois sont très différentes de celles de souvent. Notamment, étant basiquement existentiel (intersectif), parfois se combine aisément avec un indéfini objet.

(9) a. ??Un chasseur tue souvent/toujours un lapin.

b. Un chasseur tue parfois un lapin.

\footnotetext{
${ }^{3}$ La portée de jamais sur l'indéfini transforme celui-ci en un terme de polarité négative: Pierre ne boit jamais une goutte. Rarement, dont la négativité est plus faible, ne permet que des expressions de polarité négative de type J'ai rarement vu une femme aussi belle, autorisées aussi par jamais.

4 Tout comme le quantificateur nominal quelques en position accentuée (QUELques étudiants sont restés (= certains étudiants)), parfois peut avoir une lecture forte (proportionnelle) si l'ensemble des situations de référence est introduit explicitement.
} 
Les seuls Q-adverbes dont la combinaison avec un indéfini objet pose problème sont donc les quantificateurs forts souvent et toujours. Toujours établit une relation entre deux ensembles en effectuant une quantification universelle. Souvent est à la base un quantificateur proportionnel, c'est-à-dire un quantificateur qui établit la proportion des événements sur lesquels il quantifie par rapport à un ensemble de référence. Nous adopterons cependant la position défendue par de Swart (1991) selon laquelle sowvent peut aussi avoir une lecture faible, c'est-à-dire existentielle, ou (quasi-) intersective (cf. aussi Abeillé et al., 2004). Sowvent s'assimile à cet égard au quantificateur nominal beancoup de, qui se caractérise lui aussi par cette ambiguïté (cf. entre autres Kleiber, 2001) $)^{5}$. Par exemple, dans Pierre va souvent au cinéma, souvent est une lecture (quasi-)intersective, dans laquelle il n'établit (éventuellement) une relation que par rapport à toutes les autres activités de Pierre ou par rapport à la fréquence moyenne de l'activité en question dans un groupe de référence. Par contre, dans Quand Pierre va au cinéma, il mange sowvent une glace, la lecture de sowvent est proportionnelle $:$ la fréquence des situations dans lesquelles Pierre mange une glace est établie par rapport à l'ensemble des situations dans lesquelles Pierre va au cinéma.

\section{La structure catégorique et le topique indéfini}

\subsection{Structuration de l'information: approche informationnelle et approche illocutoire}

Selon Kuroda (1973), une phrase catégorique est une structure binaire dans laquelle le constituant initial (souvent le SN sujet) joue le rôle de topique (au sens de ce dont on parle) et le reste de la phrase, qui correspond au SV dans le cas classique, constitue le focus (ce que l'on dit à propos du topique). Cette structuration binaire est opposée à la structure thétique, qui est une structure monopartite, dépourvue de topique ${ }^{6}$.

Il faut noter que, dans la tradition anglo-saxonne, le terme de focus dénote deux phénomènes assez différents. Lorsqu'il est utilisé au sein du couple topique-focus, il dénote l'une des deux composantes de la structure catégorique, où il est équivalent au second terme du couple thème-rhème, issu de l'Ecole de Prague, ou des couples thèmepropos ou thème-commentaire dans la tradition linguistique française. D'autre part, le terme de focus désigne aussi le marquage prosodique de l'élément le plus saillant, sur le plan pragmatique ou sémantique, de l'énoncé. Utilisé dans ce second sens, ce terme désigne donc aussi bien une caractéristique prosodique qu'une caractéristique pragmatique/ sémantique (cf. par exemple Rooth, 1996).

\footnotetext{
${ }^{5}$ Le relecteur anonyme suggère que la contrepartie nominale de souvent est la plupart (de) (plus de la moitié). La raison principale qui nous fait écarter cette option est que la plupart est un quantificateur purement proportionnel, qui ne permet pas de lecture intersective. Beaucoup (de) est employé comme contrepartie de souvent par ex. dans Abeillé et al. (2004). Dans des analyses basées sur l'anglais, most ('la plupart') est utilisé comme contrepartie de usually ('généralement') (cf. entre autres Rooth, 1995 ; Chierchia, 1995 ; Krifka, 2001).

${ }^{6}$ Cohen \& Erteschik-Shir (2002) avancent des arguments qui tendent à prouver que toutes les phrases ont un topique et que la particularité des phrases thétiques ne consiste qu'en ce que leur topique est implicite (inféré du contexte discursif ou situationnel).
} 
Svetlana Vogeleer

Dans certaines approches qui mettent l'accent sur la relation entre le contour prosodique de l'énoncé et les actes illocutoires, la segmentation binaire de la phrase en topique (ce dont on parle), et focus (ce que l'on dit à propos du topique) est remplacée par (ou coexiste avec) la structuration fond-focus, qui se situe sur le plan illocutoire (Jacobs, 1991 ; Beyssade et al., 2004 ; Marandin, 2006). Le terme de fond dénote la partie de l'énoncé qui ne fait pas (plus) l'objet de l'acte illocutoire parce qu'elle contient une information inférable du contexte, et celui de focus dénote la partie qui constitue l'enjeu d'un acte illocutoire (assertion, question, ordre) (Beyssade et al., 2004).

Dans le cadre de cette recherche, nous adopterons une position selon laquelle la distinction entre les deux approches n'est pas très pertinente en ce qui concerne la structure catégorique de base, caractéristique des assertions simples (ex. 1-3), puisque le topique de la structure catégorique correspond au fond dans l'approche illocutoire, et le focus est, dans les deux approches, un focus large, qui correspond à l'ensemble du SV. Par contre, lorsque un seul élément de la phrase est marqué par un focus étroit, notamment par un focus contrastif, c'est la structuration illocutoire fond-focus, plus souple, qui semble être mieux adaptée pour rendre compte du contenu sémanticopragmatique en fonction des connexions discursives de l'énoncé.

\subsection{Anaphoricité de l'indéfini sujet générique}

Une structure informationnelle n'est pas interprétée de manière autonome, mais par rapport à un énoncé antécédent ${ }^{7}$, explicite ou reconstruit au cours de l'interprétation. Certaines structures permettent de reconstruire plusieurs énoncés antécédents possibles, de sorte que l'interprétation varie en fonction de l'antécédent attribué. Une structure peut aussi avoir un antécédent vide. Cela ne signifie pas qu'elle soit insensible à tout antécédent, mais qu'elle peut être employée out of the blue (mais voir note 6).

Au premier abord, la structure catégorique $\left[\mathrm{Un} \mathrm{N}_{\text {suj }}\right]_{\mathrm{T}}\left[\mathrm{V}_{\mathrm{tr}} \mathrm{Q}-\mathrm{adv} \text { un } \mathrm{N}_{\text {ов }}(\mathrm{X})\right]_{\mathrm{F}}$ des phrases génériques semble être dépourvue de tout antécédent parce que son sujet est un indéfini. Il est cependant peu probable qu'un professeur énonce (10a) à peine entré en classe, sans un préambule comme celui de (10b) :

(10) a.[Un adjectif $]_{\mathrm{T}}[$ suit souvent un nom] $\mathrm{F}$.

b. Aujourd'hui, nous parlerons des adjectifs. [Un adjectif $]_{\mathrm{T}}$ [suit souvent un nom] $]_{\mathrm{F}}$.

On constate, en effet, que le sujet indéfini générique de la structure catégorique requiert un antécédent (Vogeleer \& Tasmowski, 2005). Normalement, son antécédent est un défini pluriel générique (ex. 10b). Dans certains cas, l'antécédent direct est un autre indéfini générique ; c'est alors celui-ci qui est rattaché au défini pluriel générique.

Dans les grandes lignes, le défini pluriel générique dénote un ensemble maximal d'individus localisés dans tous les mondes possibles, réels, virtuels et contrefactuels, qui satisfont le contenu descriptif du nom (Kleiber, 1986, p. 67). Cela revient à dire que le défini pluriel générique dénote l'ensemble des individus qui constituent une

${ }^{7}$ Le terme d'antécédent ne dénote pas ici une expression précédente par rapport à laquelle une expression anaphorique est coréférentielle, mais un énoncé précédent, explicite ou reconstruit, par rapport auquel une structure informationnelle est interprétée. 
espèce, ou une catégorie. Étant donné que le défini pluriel générique n'introduit pas d'individus spécifiques, l'indéfini singulier générique n'est pas vraiment anaphorique, en ce sens qu'il n'est pas coréférentiel par rapport au défini.

La lecture quasi-anaphorique de l'indéfini générique s'harmonise bien avec son rôle de topique au sein de la structure catégorique, puisqu'un élément topical contient normalement une information non nouvelle, introduite préalablement. En se fondant sur ces considérations, Kuroda (1973, p. 102) estime qu'un indéfini ne peut être générique que lorsqu'il constitue le topique d'un énoncé catégorique ${ }^{8}$. S'appuyant sur Kuroda, Jonasson (1986, p. 338) développe cette idée en l'appliquant à l'indéfini en position d'objet, qui, selon elle, ne peut être générique que lorsqu'il est thématique (topical). Nous verrons plus loin qu'aussi bien la généricité que la topicalité de l'indéfini objet sont beaucoup moins nettes que pour l'indéfini sujet: d'une part, un indéfini objet générique est existentiel à la base; d'autre part, faisant partie du SV, il fait partie du focus large au niveau de la structure informationnelle.

\section{Le domaine de quantification des Q-adverbes : quantification sur les situations}

Dénotant une relation entre deux ensembles, un quantificateur fort (non intersectif) crée une structure quantificationnelle tripartite composée du quantificateur lui-même, de sa restriction, qui dénote le domaine sur lequel il quantifie, et de sa portée nucléaire. Un quantificateur nominal (quelques $N$, chaque $N$, etc.) est lié syntaxiquement au nom. C'est pourquoi son domaine de quantification par défaut est dénoté par le nom (avec ses modifieurs éventuels), le reste de la phrase se retrouvant dans la portée nucléaire. Dans (11), le quantificateur nominal quantifie sur le domaine les renards; il établit que les individus qui sont rusés (le prédicat introduit dans la portée nucléaire) représentent une proportion importante (beaucoup) d'individus de ce domaine :

(11) a. [Beaucoup de renards $]_{\mathrm{T}}$ [sont rusés $]_{\mathrm{F}}$.

b. Beaucoup de [les) renards $]_{\text {RESTr. }}$ [sont rusés $]_{\text {PORTÉE NUCL. }}$

Quant aux quantificateurs adverbiaux, leur relation syntaxique avec le domaine sur lequel ils quantifient n'est pas spécifiée. Un Q-adverbe peut être un modifieur du $\mathrm{SV}$ ou un opérateur phrastique. Cela ne pose pas trop de problèmes lorsque les $\mathrm{SN}$ de la phrase sont dépourvus de toute variabilité quantificationnelle (c'est le cas des noms propres et des définis singuliers). Par contre, lorsque la phrase comprend un SN indéfini, qui se caractérise par une variabilité quantificationnelle, cela provoque des ambiguités de portée. Par exemple, c'est la variabilité quantificationnelle de l'indéfini qui lui permet de dénoter différentes pommes en (12):

(12) Je mange souvent une pomme au petit déjeuner.

\footnotetext{
${ }^{8}$ Cohen (2001) defend un point de vue radicalement opposé, selon lequel une phrase avec un sujet indéfini générique est une structure thétique qui constitue un commentaire par rapport à une règle implicite pré-établie.
} 
Svetlana Vogeleer

L'idée que les Q-adverbes quantifient sur les situations est due à Kratzer (1989), qui se fonde elle-même sur Lewis (1975). Selon Lewis, les Q-adverbes sont des «lieurs non sélectifs » qui lient toutes les variables libres présentes dans la phrase. En liant des $n$-tuples de variables, les Q-adverbes quantifient, selon Lewis, sur des « cas », qui sont des $n$-tuples de participants, dont l'un est le paramètre temporel $t$. Kratzer remplace la notion de « cas », par celle de « situation », plus large, car les «situations » peuvent non seulement être dénotées par des éléments de la phrase, mais peuvent aussi être implicites. Toute situation étant localisée dans le temps, la quantification sur les situations donne lieu à une quantification sur les temps.

Selon Lewis (1975), (13a) a les mêmes conditions de vérité que (13b) parce que le prédicat statique de (13a) ne donne pas lieu à la quantification sur les temps.

(13) a. Une équation carrée a souvent deux solutions.

b. Beaucoup d'équations carrées ont deux solutions.

Dans le cas des prédicats statiques, qui ne déclenchent pas de quantification sur les temps, la quantification adverbiale est souvent représentée au moyen de la quantification nominale (cf. parmi beaucoup d'autres, Chierchia, 1995 ; Krifka, 2001). Cette conversion est d'autant plus facile que tous les Q-adverbes ont une contrepartie nominale (cf. section 2): toujours $\rightarrow>$ tout $\mathrm{N} /$ tous les $\mathrm{N} /$ chaque $\mathrm{N}$, souvent $->$ beaucoup de, rarement $\rightarrow>$ peu de, parfois $\rightarrow>$ quelques, jamais $\rightarrow>$ aucun.

Cependant, si (13b) est intuitivement interprétée comme une phrase statique, où le quantificateur nominal établit, de manière objective, la proportion des équations carrées qui ont deux solutions par rapport à l'ensemble des équations carrées, (13a) se caractérise par un certain dynamisme, qui témoigne de la présence d'une composante temporelle, et par une certaine subjectivité. Bien sûr, la question se pose de savoir comment une propriété aussi statique et objective d'une équation carrée que celle d'avoir deux solutions pourrait-elle être localisée dans le temps et acquérir une nuance subjective. Notre hypothèse à ce propos sera exposée dans la section 7.

Ici, nous passerons brièvement en revue différents modèles de la quantification sur les situations et les discussions qu'ils suscitent.

La quantification sur les situations prend normalement en compte les phrases avec des prédicats s-level ${ }^{9}$, c'est-à-dire des prédicats qui ont une localisation temporelle, ce qui donne lieu à la quantification sur les temps (mais voir de Swart, 1996). Les situations qui constituent le domaine de quantification du Q-adverbe sont généralement représentées sous la forme de subordonnées en quand ou si. Quand $p$ ou $s i$ $p$ sélectionnent une situation dénotée par $p$ (pour quand) ou l'hypothèse que $p$ (pour $s i$ ). La différence entre quand et si est la suivante. Quand $p$ exprime la présupposition que $p$,

\footnotetext{
${ }^{9} \mathrm{La}$ distinction entre les prédicats $s$ (tage)-level (prédicats localisés dans le temps) et les prédicats i(ndividual)-level (prédicats d'état) est due à Carlson (1977), selon lequel les premiers sont attribués à des «tranches » d'individu localisées dans le temps, tandis que les seconds sont attribués à l'individu dans sa globalité. Par exemple, le prédicat de Pierre travaille est interprété comme s-level (= est en train de travailler) si la phrase est vérifiée par rapport à un instant particulier, et comme i-level s'il caractérise Pierre globalement à une certaine période de sa vie.
} 
qui est accommodée au niveau global. Autrement dit, la présupposition se projette (est vraie) en dehors de la phrase ${ }^{10}$. Sip introduit lui aussi une présupposition, mais celle-ci ne se projette pas en dehors de l'apodose ${ }^{11}$.

La quantification sur les situations est souvent illustrée par des cas où la phrase ou sa présupposition fournissent un prédicat pour une subordonnée en quand ou si:

(14) a. [Un chat $]_{\mathrm{T}}[\text { atterrit toujours sur ses pattes }]_{\mathrm{F}}$.

b. Toujours [quand un chat $t_{i}$ tombe $]_{\text {RESTR. }}\left[i_{i} \text { atterrit sur ses pattes }\right]_{\text {PORTEE NUCL. }}$

(Shubert \& Pelletier, 1989)

L'exemple (14) pose la question de savoir comment on détermine le domaine, représenté dans la restriction, sur lequel quantifie le Q-adverbe.

Certains estiment que les Q-adverbes quantifient sur le topique (von Fintel, 1995 ; de Swart, 1996 ; Beaver, 2004), en précisant quelquefois qu'il s'agit bien du «topique de discours » (von Fintel, 1995). Cependant, cette dernière notion ne nous semble pas suffisamment stricte pour l'appliquer à la détermination de la restriction d'un Q-adverbe. Il est difficile de juger, d'après (14a), si la chute d'un chat est le topique du discours dont la phrase fait partie. D'autre part, si l'on prend le terme de topique au sens étroit, il est clair que le Q-adverbe de (14a) ne quantifie pas sur un ou les chat (s). Si c'était le cas, (14a) serait équivalente à (14c) :

(14) c.Tous les chats atterrissent sur leurs pattes.

La quantification sur des situations en (14b) est une quantification restreinte : le quantificateur ne s'applique qu'aux situations où un chat tombe. En (14c), le quantificateur s'applique à l'ensemble des chats, y compris à ceux qui ne tombent jamais.

Des phrases comme (14a) servent d'argument à ceux qui soutiennent que le Q-adverbe quantifie sur la présupposition, en entendant par présupposition une présupposition sémantique stricte, c'est-à-dire existentielle (cf. Shubert \& Pelletier, 1989 ; Geurts \& van der Sandt, 2004). En (14b), la présupposition est obtenue à partir du contenu lexical du verbe atterrir en vertu du raisonnement qu'il est nécessaire qu'un chat tombe pour qu'il puisse atterrir.

La première hypothèse est donc que le Q-adverbe quantifie sur le topique (du discours), la deuxième est que le Q-adverbe quantifie sur la présupposition, et enfin, la troisième hypothèse, soutenue dans le cadre de la sémantique du focus (Rooth, 1995 ; 1996 ; 1999 ; Cohen 2003 ; 2004), est que le Q-adverbe quantifie sur des «alternatives»,

10 Selon van der Sandt (1992), les présuppositions sont des propositions anaphoriques, qui ont un antécédent explicite ou implicite. Lorsque l'antécédent est implicite, la présupposition est accommodée, ce qui signifie que son antécédent est reconstruit au cours de l'interprétation. On parle d'une accommodation globale si l'antécédent est reconstruit en dehors de la phrase qui contient la présupposition. Si l'antécédent est reconstruit au sein de la même phrase que la présupposition, l'accommodation est intermédiaire ou locale.

${ }^{11}$ Cette différence entre quand et si n'est pas toujours perceptible, mais elle apparait clairement si l'on compare (i) Quand les chats naissent, ils sont aveugles et (ii) Si les chats naissent, ils sont aveugles. En (i), la naissance des chats est une présupposition qui est accommodée au niveau global, c'est-à-dire est vraie en dehors de la phrase. En (ii), la présupposition ne se projette pas. L'accommodation est ici intermédiaire, car la vérité de la protase n'est valable que pour les mondes décrits par l'apodose. 
qui sont définies comme des "présuppositions faibles » «évoquées » par l'élément focal de la phrase (Rooth, 1999). Dans le cadre de la sémantique du focus, la représentation sémantique de (14a) serait glosée par quelque chose comme (14d), où l'élément marqué par A(lternatives) dénote des alternatives au contenu descriptif de l'élément introduit par une clivée dans la portée nucléaire (c'est sur ses pattes que...). Quant au Q-adverbe, il s'associe à l'élément focal de la clivée :

d. [Quand un chati atterrit sur une partie de son corps $s^{\mathrm{A}}(\text { sur sa tête, sur son dos, ...) }]_{\mathrm{RESTR}}$. [c'est toujours sur ses pattes qu'ili atterrit $]_{\text {PORTEE NUCL. }}$

Pour déclencher des alternatives dans la restriction, le syntagme prépositionnel (SP) sur ses pattes de (14a) ne doit pas nécessairement être lié par une relation de contraste à un élément d'un énoncé antécédent. Ce SP est considéré comme l'élément le plus saillant simplement parce qu'il occupe la position finale au sein d'un SV qui correspond au focus large de la structure catégorique. Selon Kiss (1998), il s'agit alors d'un focus informationnel, qu'elle oppose au focus identificationnel (appelé aussi focus contrastif).

Dans l'analyse (14b), le prédicat de la restriction est inféré du contenu lexical du verbe de départ. Quant au Q-adverbe, il établit la proportion des situations dans lesquelles un chat atterrit sur ses pattes par rapport à l'ensemble des situations dans lesquelles un chat tombe. Dans l'analyse (14d), le domaine de quantification est plus restreint : la proportion des situations dans lesquelles un chat atterrit sur ses pattes est établie par rapport à l'ensemble des situations dans lesquelles un chat atterrit sur une partie de son corps. Pour cet exemple précis, on pourrait admettre que les deux analyses sont équivalentes en termes de conditions de vérité (quand on tombe, on atterrit inévitablement sur une partie de son corps). Cependant, ce n'est pas toujours le cas. Notre position est que la quantification en termes d'alternatives permet de circonscrire le domaine de quantification de manière plus restreinte, et donc plus précise, que l'analyse en termes de présupposition.

L'hypothèse que nous développerons dans les sections 5 et 6 pour la structure [Un $\mathrm{N}_{\text {suj] }}$ [ $\left[\mathrm{V}_{\text {tr }} \mathrm{Q}-\mathrm{adv} \text { un } \mathrm{N}_{\text {овл }}(\mathrm{X})\right]_{\mathrm{F}}$ avec un prédicat s-level est que son indéfini objet n'est acceptable sans réserves qu'à condition qu'il fasse partie de la restriction soit luimême, soit sous la forme d'alternatives qu'il déclenche. Si l'indéfini objet se retrouve dans la portée nucléaire sans avoir un antécédent dans la restriction, il déclenche une quantification existentielle qui suspend sa variabilité quantificationnelle.

\section{L'indéfini objet en position non finale}

Condoravdi (1989) constate que les phrases avec des verbes moyens sont ressenties comme «défectueuses» si leur verbe n'est pas suivi d'un modifieur (un adverbe de manière) ou d'un ajout (un syntagme prépositionnel) :

(15) a. ??Une tulipe pousse.

a'. Une tulipe pousse vite/dans un sol sablonneux.

b. ?? Un Espagnol parle.

b'. Un Espagnol parle vite. 
Condoravdi avance l'hypothèse que l'élément postverbal est requis par la structure quantificationnelle: le prédicat de ces verbes produit un quantificateur générique qui nécessite une restriction. Grâce à l'élément postverbal, la prédication de la phrase (le verbe et son sujet) forme la restriction, tandis que l'élément postverbal va dans la portée nucléaire. L'absence d'élément postverbal empêche la mise en place de cette structure quantificationnelle.

Dans la structure [Un $\left.\mathrm{N}_{\text {suj }}\right]_{\mathrm{T}}\left[\mathrm{V}_{\mathrm{tr}} \mathrm{Q} \text {-adv un } \mathrm{N}_{\text {ов }}(\mathrm{X})\right]_{\mathrm{F}}$, le rôle de l'élément $\mathrm{X}$ est similaire à celui de l'adverbe ou du SP dans les exemples (15). Quand l'indéfini objet est suivi d'un modifieur, nominal ou verbal, ou d'un SP, cet élément postposé assume par défaut la fonction de focus informationnel au sein du focus large (le SV). Par conséquent, c'est cet élément qui va dans la portée nucléaire, tandis que la restriction sera formée par la structure syntaxique $\mathrm{S}-\mathrm{V}_{\mathrm{tr}} \mathrm{O}$.

Cette procédure explique la différence d'acceptabilité entre (16a) et (16b) (voir aussi (3) et (2)). Grâce au modifieur postposé, en l'occurrence une relative, (16a) autorise la quantification (16c). Par contre, en (16b), où l'indéfini objet ne contient qu'un nom, c'est cet indéfini «nu » qui constitue le focus informationnel par défaut et qui devrait, de ce fait, figurer dans la portée nucléaire. Or, les éléments restants, $\mathrm{S}-\mathrm{V}_{\mathrm{tr}}$ (quand un castor construit), ne forment pas une structure syntaxique complète apte à décrire une situation dans la restriction.

(16) a.[Un castor $]_{\mathrm{T}}$ [construit souvent un barrage qui résiste à toutes les intempéries] $\mathrm{F}$. b. ??[Un castor $]_{\mathrm{T}}$ [construit souvent un barrage $]_{\mathrm{F}}$.

c. Souvent [quand un castor $\mathrm{i}_{\mathrm{i}}$ construit un barrage $]_{\mathrm{RESTR}}$. [i $\mathrm{i}_{\mathrm{i}}$ construit [un barrage [qui résiste à toutes les intempéries]]]j porTEE NucL.

En (16c), le barrage qu'un castor construit est recatégorisé dans la portée nucléaire sous un prédicat plus spécifique qui dénote une sous-catégorie de barrages. Suite à cette recatégorisation, l'indéfini modifié de la portée nucléaire "évoque des alternatives » dans la restriction (Rooth 1999). Ces alternatives sont ici d'autres souscatégories de barrages établies selon le critère de solidité. En fin de compte, cela donne une représentation conforme au modèle de Rooth (ex. 16d), où la portée nucléaire contient une clivée :

(16) d. [Quand un castor construit un barrage $\left.{ }^{\mathrm{A}}\right]_{\text {RESTR. }}$ [c'est souvent un barrage qui résiste à toutes les intempéries qu'il construit $]_{\text {PORTEE NUCL. }}$

La structure quantificationnelle sera similaire à (16c, d) si l'élément postposé est un modifieur adverbial ou un SP.

La restriction de (16c) est obtenue au moyen de la soustraction du focus informationnel. Cette restriction ne correspond pas au topique (le SN sujet) de la structure catégorique. D'autre part, elle ne représente pas nécessairement le topique du discours : le discours précédent ne doit pas obligatoirement porter sur la construction de barrages (par un castor). Selon Rooth (1999), une restriction obtenue au moyen de la soustraction du focus étroit (un focus informationnel ou un focus contrastif) peut être une présupposition existentielle ou une " présupposition faible » (cf. section 4). En (16a), les situations dans lesquelles un castor construit un barrage qui résiste à toutes les intempéries ne pourraient pas exister s'il n'existait pas de situations dans lesquelles 
un castor construit un barrage. Cela signifie que la restriction en (16c) est bien une présupposition existentielle. C'est pourquoi la lecture du Q-adv souvent est ici proportionnelle (forte) : sowvent établit la proportion des situations dans lesquelles un castor construit un barrage d'un certain type par rapport à l'ensemble des situations dans lesquelles un castor construit un barrage.

Comparons à cet égard (16a) à (17a).

(17) a. [Un requin $]_{\mathrm{T}}^{\mathrm{T}}$ [attaque souvent un baigneur blessé $]_{\mathrm{F}}$.

b. ? Un requin] $]_{\mathrm{T}}$ [attaque souvent un baigneur] ${ }_{\mathrm{F}}{ }^{12}$.

La phrase (17a) permet une lecture proportionnelle du Q-adverbe, similaire à celle de (16c) : sowvent établit la proportion des situations dans lesquelles un requin attaque un baigneur blessé par rapport à l'ensemble des situations dans lesquelles un requin attaque un baigneur :

(17) c. Souvent [quand un requin ${ }_{i}$ attaque un baigneur $]_{\mathrm{RESTR}}$. [i $\mathrm{i}_{\mathrm{i}}$ attaque [un baigneur [blessé]]]] PORTEE NUCL.

d. [Quand un requin attaque un baigneur $\left.{ }^{\mathrm{A}}\right]_{\mathrm{RESTR}}$. [c'est souvent un baigneur blessé qu'il attaque] $]_{\text {PORTEE NUCL. }}$

Cependant, (17a) permet aussi une lecture quasi-intersective du Q-adverbe, décrite en (17e).

(17) e. Souvent [quand un requin ${ }_{i}$ est confronté à un baigneur [et que ce baigneur $_{j}$ est blessé]] $]_{\text {RESTR. [ili }}$ [attaque] [un baigneur blesséj]j pOrTÉE nucL.

La restriction de (17e) contient un verbe qui dénote une relation de proximité spatiale. Cette relation est une présupposition produite par la phrase: un requin ne peut attaquer quelqu'un qu'à condition qu'il se trouve à une distance qui rend l'objet de son attaque accessible. Cette condition pourrait être rendue par tout autre verbe qui décrit l'accessibilité de l'objet, par exemple voir un baigneur blessé. Suite à la substitution du verbe, c'est le verbe de départ qui se retrouve dans la portée nucléaire, tandis que l'indéfini objet, avec son modifieur, va dans la restriction. Le modifieur joue ici le rôle d'un prédicat qui restreint davantage le domaine de quantification. A la différence de la lecture (16c), la proportion des situations dans lesquelles un requin attaque un baigneur blessé n'est pas établie par rapport à l'ensemble des situations dans lesquelles un requin attaque un baigneur, mais par rapport à l'ensemble des situations dans lesquelles un requin est confronté à un baigneur blessé. Les attaques contre d'autres catégories de baigneurs (ceux qui ne sont pas blessés) ne sont pas prises en compte.

Bien que le Q-adverbe établisse une proportion par rapport aux situations décrites dans la restriction, cette lecture de sowvent est pratiquement intersective (faible), puisqu'un requin n'a aucun moyen d'attaquer qui que ce soit si l'individu en question se trouve hors de son atteinte.

12 Bien que l'indéfini objet de (17b) ne comporte pas de modifieurs, cette phrase est acceptée plus facilement que, par exemple, ??Un requin attaque souvent un homme. La raison en que que le nom un baigneur est suffisamment spécifique pour déclencher des alternatives par rapport à d'autres souscatégories d'humains (des non-baigneurs) (cf. section 6). 
A la différence de la lecture (17c), le modifieur en (17e) ne déclenche pas d'alternatives parce qu'il fait partie de la restriction. Cependant, cela ne signifie pas qu'il soit sémantiquement superflu et qu'un nom «nu » puisse aller dans la restriction tout aussi facilement. Au sein de la restriction, le modifieur se présente comme un prédicat qui ajoute une restriction supplémentaire. Cette opération déclenche une recatégorisation dans la portée nucléaire, qui permet de passer de la prédication le baigneur (auquel un requin est confronté) EST blessé au SN un baigneur blessé. En l'absence de modifieur, la quantification (17e) ne serait pas possible.

Dans la lecture (17e), le verbe est le seul élément non anaphorique de la portée nucléaire. S'il se trouve dans la portée nucléaire ce n'est pas parce qu'il porte un focus contrastif, mais uniquement parce qu'il ne fait pas partie de la restriction. Ce cas diffère de celui où le verbe porte un focus contrastif. Le focus contrastif sur le verbe rend acceptable tout indéfini objet non modifié parce que celui-ci va dans la restriction.

(18) a. ? [Un requin $]_{\mathrm{T}}[\text { attaque souvent un baigneur }]_{\mathrm{F}}$.

b. A. Un requin s'enfuit quand il voit un baigneur. - B. Mais non! Un requin [ATTAQUE] $]_{F C}$ un baigneur.

Dans les deux cas examinés ici, l'indéfini sujet se caractérise par une variabilité quantificationnelle due à une restriction en quand combinée au présent du verbe. Quant à l'indéfini objet, notre analyse mène à la conclusion qu'il manifeste une variabilité quantificationnelle lorsqu'il se trouve lui aussi dans la restriction. Comme nous le verrons dans la section suivante, l'indéfini objet manifeste aussi une variabilité quantificationnelle lorsqu'il est représenté dans la restriction par un antécédent.

\section{L'indéfini objet en position finale : prédicats s-level}

L'énoncé antécédent de (19a) est tel qu'il ne déclenche pas d'opposition entre construire un barrage et construire tout autre objet alternatif (un mur, une maison, etc.). Par conséquent, l'indéfini objet forme avec le verbe un groupe focal indivisible et la phrase n'offre pas de prédicat pour construire une restriction en quand. On pourrait, bien sûr, suppléer pour la restriction une description aussi large que possible, telle que (construire) quelque chose ou (construire) un ouvrage (ex. 19b), mais, en l'absence de toute opposition, cette solution ne correspond pas à la lecture par défaut.

(19) (Aujourd'hui, nous parlerons des castors).

a. ??[Un castor $]_{\mathrm{T}}$ [construit toujours un barrage $]_{\mathrm{F}}$.

b. ??Toujours [quand un castor $\mathrm{i}_{\mathrm{i}}$ construit quelque chose/un ouvrage $]_{\text {RESTR. }}\left[\mathrm{i}_{\mathrm{i}}\right.$ construit un barrage] $]_{\text {PORTEE NUCL. }}$

Par contre, si la phrase est reliée à un énoncé antécédent comme celui de (20), l'indéfini objet sera marqué par un focus contrastif. Le focus contrastif déclenche une opposition entre un barrage et la catégorie mentionnée dans l'énoncé antécédent. De manière plus large, tout antécédent qui déclenche l'opposition de un barrage à d'autres catégories alternatives permet d'inférer un prédicat commun à toutes ces catégories (ici un owvrage), et c'est ce prédicat qui va dans la restriction (ex. 20b). Cette procédure 
assure en même temps un prédicat pour l'indéfini sujet et un antécédent pour l'indéfini objet. Le fait d'avoir un antécédent dans la restriction garantit la variabilité quantificationnelle, tandis que l'indéfini de la portée nucléaire ne fait que recatégoriser le référent sous un autre nom.

(20) (Mais non, mon chéri, ce castor ne construit PAS une maison.)

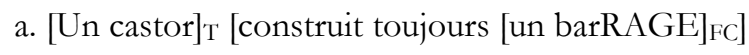

b. Toujours $\left[\text { quand un castor }{ }_{i} \text { construit un ouvrage }{ }^{\mathrm{A}}\right]_{\mathrm{RESTR}}$. $\left[\mathrm{i}_{\mathrm{i}}\right.$ construit $[\mathrm{un}$ barrage]] PORTEE NUCL.

Les alternatives peuvent être introduites explicitement (ex. 21) (Rooth 1996, 1999 ; Cohen 2003). Dans ce cas, on infère de l'ensemble des alternatives un prédicat commun, un animal en (21b), qui sera repris dans la restriction. L'indication A(lternatives) signale que ce prédicat ne dénote pas une entité qui satisfait au prédicat être un animal, mais une espèce d'animaux qui sera opposée à d'autres espèces dans la portée nucléaire. La liste d'alternatives peut être close ou ouverte :

(21) a.??Un chasseur tue souvent un lapin.

b. [Un chasseur] $]_{\mathrm{T}}$ [tue souvent un lapin, un renard ou une perdrix (et peut-être d'autres animaux encore)] F.

Souvent [quand un chasseur tue un animal $\left.{ }^{\mathrm{A}}\right]_{\mathrm{RESTR}}$. [il tue [un lapin, un renard ou une perdrix]] PORTEE NUCL.

Les particules focalisantes aussi et même déclenchent elles aussi des alternatives (cf. Cohen 2004) parce qu'elles présupposent qu'il y a un complémentaire de l'ensemble dénoté par l'indéfini :

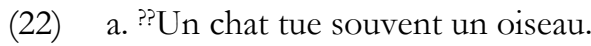

b. Les chats sont méchants! Un chat tue parfois même un oiseau !

La phrase (21a) a deux défauts : d'une part, son indéfini sujet n'a pas de prédicat qui puisse être placé dans la restriction, et d'autre part, son indéfini objet n'a pas d'antécédent dans la restriction. C'est pour ces deux raisons que les exemples en (23) semblent bizarres dans un contexte neutre qui ne déclenche pas d'alternatives.

(23) a. ?? Un chat mange souvent une saucisse.

b. ??Un requin attaque toujours un homme.

c. ??Un chasseur tue souvent un lapin. 


\section{L'indéfini objet en position finale : prédicats i-level}

Contrairement à la série (23), l'acceptabilité des phrases en (24) ne suscite pas objections.

(24) a. [Un adjectif $]_{\mathrm{T}}$ [suit souvent un nom $]_{\mathrm{F}}$.

b. [Un cowboy $]_{\mathrm{T}}$ [porte toujours un revolver $]_{\mathrm{F}}$.

c. [Un Cubain $]_{\mathrm{T}}[\text { fume souvent un cigare }]_{\mathrm{F}}$.

Notons que ces phrases permettent plus d'une lecture. Elles peuvent notamment être interprétées en termes d'alternatives au nom objet. Par exemple, (24c) permet la lecture (25) en termes d'alternatives à fumer un cigare (fumer la pipe, fumer des cigarettes,...). Dans l'analyse qui suit, nous ne tiendrons pas compte de cette interprétation.

(25) [quand un Cubaini fume un produit fumable $\left.{ }^{\mathrm{A}}\right]_{\mathrm{RESTR}}$. [c'est souvent un cigare qu'il fume] $]_{\text {PORTEE NUCL. }}$

Les phrases (24) peuvent aussi être interprétées par rapport à une question de type $A$ quoi reconnait-on un Cubain/un cowboy? ou Quelle est la propriété distinctive d'un adjectif? C'est à cette lecture que nous nous intéresserons ici.

Dans la lecture en termes de propriété distinctive, le SV est interprété comme un prédicat i(ndividual)-level, c'est-à-dire comme statique, bien qu'il puisse contenir des verbes qui permettent aussi une lecture s-level (ex. 24b, 24c). Comme le montre (25), la lecture s-level du prédicat est associée à la lecture en termes d'alternatives, où le Q-adverbe force le prédicat de la restriction à dénoter des situations localisées dans le temps.

Si dans un contexte de type $A$ quoi reconnaît-on un Cubain/un cowboy? le prédicat est interprété comme i-level, cela signifie que ni le verbe ni l'indéfini objet ne font partie de la restriction. Naturellement, la question se pose de savoir ce que contient la restriction du Q-adverbe, puisque, d'une part, celui-ci force la localisation temporelle des situations sur lesquelles il quantifie, mais d'autre part, dans la lecture qui nous intéresse, le prédicat est statique.

Comme nous l'avons mentionné dans la section 4, Lewis (1975), ainsi que beaucoup d'autres auteurs, estiment que, pour les phrases avec un prédicat statique, la quantification adverbiale se ramène à la quantification nominale (ex. (13), repris cidessous sous (26)).

(26) a. Une équation carrée a souvent deux solutions.

b. Beaucoup d'équations carrées ont deux solutions.

Cependant, la perception intuitive de (26a) et (26b) n'est pas identique. La phrase (26a) est ressentie comme plus dynamique et plus subjective que (26b), qui attribue, de manière objective, une propriété statique à une partie (importante) de l'ensemble des équations carrées.

L'analyse (27) que nous proposons rend compte du dynamisme et de la subjectivité de (26a). 
(27) Souvent [quand on tombe sur/on a affaire à une équation carrée $]_{\mathrm{RESTR}}$. [cette équation carrée a deux solutions] PORTÉE NUCL.

En (27), le Q-adverbe quantifie sur des situations dans lesquelles un agent universel (on) est lié par une relation de contact aléatoire (on tombe sur/on a affaire àlon est confronté a) au référent de l'indéfini sujet. Dans la portée nucléaire, le référent de l'indéfini sujet se voit attribuer la propriété dénotée par le prédicat.

L'avantage de cette analyse est double. D'une part, la restriction de (27) satisfait à la condition de localisation temporelle imposée par l'adverbe, ce qui rend compte du dynamisme, c'est-à-dire de la quantification sur les temps, qui distingue (26a) de (26b). D'autre part, l'introduction de l'agent universel on rend compte de la subjectivité (une information basée sur l'expérience) qui oppose (26a) à l'information objective (une information basée sur la connaissance) au sujet d'un sous-ensemble d'équations carrées exprimée au moyen de (26b).

Selon Lewis (1975) et autres auteurs qui représentent la quantification adverbiale au moyen de la quantification nominale (cf. section 4), (26a) a les mêmes conditions de vérité que (26b). Notre analyse (27) ne garantit pas l'équivalence vériconditionnelle entre (26a) et (26b). Une relation de contact aléatoire (quand on tombe sur/on a affaire a) ne garantit pas que chaque situation contienne un référent différent. Or, comme on peut tomber plusieurs fois sur le même référent, souvent ne garantit pas à $100 \%$ que les équations carrées qui ont deux solutions constituent, par exemple, plus de $50 \%$ de l'ensemble des équations carrées, même si cette inférence reste plausible.

Notons que la reprise anaphorique de l'indéfini sujet dans la portée nucléaire n'est pas effectuée en (27) au moyen d'un pronom personnel, comme c'était le cas dans les analyses présentées dans la section 5, mais au moyen d'un SN démonstratif. La raison en est la suivante. Dans les phrases examinées dans la section 5 , l'indéfini sujet recevait un prédicat dans la restriction. Or, l'attribution d'un prédicat permet d'identifier le référent par la suite, ce qui autorise une anaphore pronominale. En (27), l'indéfini, qui apparait dans la restriction sous la forme d'un complément d'objet, ne reçoit pas de prédicat susceptible d'identifier son référent par la suite. C'est pourquoi la référence anaphorique dans la portée nucléaire ne peut être assurée qu'au moyen d'un démonstratif.

L'analyse (27) s'applique aux phrases (24), qui ne diffèrent de (26a) qu'en ce que leur $\mathrm{SN}$ objet est un indéfini singulier :

(28) a. Souvent [quand on tombe sur/on voit un adjectif $]_{\text {RESTR. }}$ [cet adjectif suit un nOm] PORTEE NUCL.

b. Toujours [quand on tombe sur/on voit un cowboy] $]_{\text {RESTR. }}$. [ce cowboy porte un revolver] $]_{\text {PORTEE NUCL. }}$

c. Souvent [quand on tombe sur/on voit un Cubain $]_{\text {RESTR. [ce Cubain fume }}$ un cigare $]_{\text {PORTEE NUCL. }}$

Si les phrases (24) sont ressenties comme vraies et non problématiques c'est parce que leur prédicat permet une lecture statique en termes de propriété distinctive. Si l'on accepte comme vrai que chaque fois qu'un agent quelconque tombe sur un cowboy, celui-ci porte un revolver, c'est parce qu'on interprète la propriété de porter 
un revolver comme une propriété distinctive, et donc statique et permanente, d'un cowboy, et non pas comme une quantification sur des situations dans lesquelles un cowboy porte un revolver.

C'est cette possibilité d'une lecture statique du prédicat en termes de propriété distinctive qui distingue les phrases en (24) de celles en (23), reprises sous (29) cidessous. Le prédicat de ces phrases peut difficilement être interprété comme i-level. C'est pourquoi elles apparaissent comme fausses, et donc bizarres, sous l'analyse en question.

(29) a. ?? Un chat mange souvent une saucisse.

a'. Souvent [quand on tombe sur un chat $]_{\text {RESTR. }}$ [ce chat mange (= est en train de manger) une saucisse] PORTEE NUCL.

b. ??Un requin attaque toujours un homme.

b'. Toujours [quand on tombe sur un requin] $]_{\text {RESTR. }}$ [ce requin attaque (= est en train d'attaquer) un homme] $]_{\text {PORTEE NUCL. }}$

c. ??Un chasseur tue souvent un lapin.

c'. Souvent [quand on tombe sur un chasseur $]_{\mathrm{RESTR}}$. [ce chasseur tue $(=$ est en train de tuer) un lapin] PORTEE NucL.

Ajoutons que l'adverbe parfois autorise cette lecture aussi bien avec des prédicats s-level qu'avec des prédicats i-level :

(30) a. Un chat mange parfois un saucisse.

a'. Parfois [quand on tombe sur un chat $]_{\text {RESTR. }}$ [ce chat mange (= est en train de manger) une saucisse] PORTEE NUCL. $_{\text {. }}$

b. Un chat est parfois dangereux.

b'. Parfois [quand on tombe sur un chat $]_{\text {RESTR. }}$ [ce chat est dangereux $]_{\text {PORTEE NUCL. }}$

\section{Conclusion}

Notre étude montre que l'indéfini objet des phrases génériques avec une structure catégorique de base n'est problématique en position finale qu'avec les Q-adverbes souvent et toujours. Les autres Q-adverbes, notamment rarement et parfois, autorisent l'indéfini objet en position finale. Les raisons de cette association varient selon l'adverbe.

L'étude des phrases avec les Q-adverbes sowvent et toujours permet de conclure que, lorsque la phrase contient un prédicat s-level, l'indéfini objet ne pose pas de problèmes d'interprétation, et donc d'acceptabilité, s'il fait partie de la restriction du Qadverbe soit lui-même soit sous la forme d'un antécédent qui dénote des alternatives. Cette condition, qui assure la variabilité quantificationnelle de l'indéfini, est satisfaite si le nom objet est suivi d'un modifieur, verbal ou nominal, ou d'un syntagme prépositionnel ou si le nom indéfini en position finale déclenche des alternatives sous la forme d'un antécédent de l'indéfini dans la restriction.

L'indéfini objet en position finale est autorisé par les prédicats i-level ou par des prédicats interprétés comme i-level. Nous proposons pour ces phrases une analyse en termes de quantification sur les situations qui diffère radicalement de l'analyse 
traditionnelle en termes de quantification nominale. Dans la solution proposée, le référent de l'indéfini sujet est introduit au moyen d'une relation de contact aléatoire avec l'agent universel on. Cette solution offre l'avantage de rendre compte à la fois de la stativité du prédicat, de la localisation temporelle des situations sur lesquelles quantifie le Q-adverbe et de la subjectivité qui caractérise la quantification adverbiale.

\section{REFERENCES}

Abeille, A. eT AL., 2004, Adverbs and quantification, in F. Corblin \& H. de Swart (eds.), Handbook of French Semantics, CSLI Publications, Stanford, p. 185-209.

BARWISE, J. \& COOPER, R., 1981, Generalized quantifiers and natural languages, Linguistics and Philosophy, 4, P. 159-219.

Beaver, D., 2004, Accommodating topics, in H. Kamp \& B. Partee (eds.), Context-Dependence in the Analysis of Linguistic Meaning, Elsevier, Amsterdam, p. 79-90.

BEyssade, C. ET AL., 2004, Prosodic, syntactic and pragmatic aspects of information structure - an introduction, in F. Corblin \& H. de Swart (eds.), Handbook of French Semantics, CSLI Publications, Stanford, p. 455-475.

BONAMI, O. ET AL., 2004, Adverb classification, in F. Corblin \& H. de Swart, (eds.), Handbook of French Semantics, CSLI Publications, Stanford, p. 143-184.

Carlson, G., 1977, A unified analysis of the English bare plural, Linguistics and Philosophy, 1, p. $413-457$.

Chierchia, G., 1995, Individual-level predicates as inherent generics, in G. Carlson \& F. Pelletier (eds.), The Generic Book, University of Chicago Press, Chicago, p. 176-223.

CoHen, A., 2001, On the generic use of indefinite singulars, Journal of Semantics, 18, p. 183- 209.

CoHen, A. 2003, How does focus affect logical form?, in Y. Falk (ed.), Proceedings of LATL 19, disponible à l'adresse http://micro5.mscc.huji.ac.il/ english/IATL/19.TOC.html

CoHen, A., 2004, Existential generics, Linguistics and Philosophy , 27, p. 137-168.

Cohen, A. \& ERTESCHIK-SHIR, N., 2002, Topic, focus, and the interpretation of bare plurals, Natural Language Semantics, 10, p. 125-165.

CONDORAVDI, C., 1989, The middle: Where semantics and morphology meet, in MIT Working Papers in Linguistics, vol. 11, MIT.

DoETJEs, J., 2002, Comparing adverbs of quantity, Ms., University of Utrecht.

von FINTEL, K., 1995, A minimal theory of adverbial quantification, in H. Kamp \& B. Partee (eds.), Proceedings of the Workshops in Prague and Bad Teinach, February 1995, vol. 1, Stuttgart University Working Papers, Stuttgart, p. 153-193.

GEURTS, B. \& VAN DER SANDT, R., 2004, Interpreting focus, Theoretical Linguistics, 30(1), p. 1-44. 
JACOBS, J., 1991, Focus ambiguities, Journal of Semantics, 8, p. 1-36.

JONASSON, K., 1986, L'article indéfini générique et la structure de l'énoncé, Travaux de linguistique et de littérature, 24(1), p. 309-345.

KISs, K. É., 1998, Identificational focus vs. informational focus, Language, 74, p. 245-273.

KLEIBER, G., 1986, Du côté de la généricité verbale: les approches quantificationnelles, Langages, 79 , p. 61- 88.

KLEIBER, G., 2001, Indéfinis : lecture existentielle et lecture partitive, in G. Kleiber, B. Laca \& L. Tasmowski (éds), Typologie des groupes nominaux, Rennes, PUR, p. 47-97.

Kratzer, A., 1989, An investigation of the lumps of thoughts, Linguistics and Philosophy, 12, p. 607-653.

KRIFKA, M., 2001, Non-novel indefinites in adverbial quantification, in C. Condoravdi \& G. Renardel de Lavalette (eds.), Logical Perspectives on Language and Information, CSLI Publications, Stanford, p. 1-40.

KuRODA S.Y., 1973, Le jugement catégorique et le jugement thétique. Exemples tirés de la syntaxe japonaise, Langages, 30, p. 81-110.

LANDMAN, F., 2006, Indefinite time phrases, in situ scope, and dual-perspective intensionality, in S. Vogeleer \& L. Tasmowski (eds), Non-definiteness and Plurality, Amsterdam, Benjamins, p. 237-266.

LEWIS, 1975, Adverbs of quantification, in E. Keenan (ed.), Formal Semantics of Natural Language, CUP, Cambridge, p. 3-15.

Marandin, J.-P., 2006, Contours as constructions, in D. Schoenefeld (ed.), Constructions, Special vol. 1, Constructions 'All Over': Case Studies and Theoretical Implications, disponible à http://www.constructions-online.de/articles/special1/

Molinier, Ch. \& Levrier, F., 2000, Grammaire des adverbes. Description des formes en ment, Droz, Genève.

RoоTH, M., 1995, Indefinites, adverbs of quantification and focus semantics, in G. Carlson \& J. Pelletier (eds.), The Generic Book, Chicago University Press, Chicago, p. 265-291.

Rooth, M., 1996, Focus, in S. Lappin (ed.), The Handbook of Contemporary Semantic Theory, Blackwell, London, p. 271-297.

Rooth, M., 1999, Association with focus or association with présupposition? In P. Bosch \& R. van der Sandt (eds.), Focus: Linguistics, Cognitive \& Computational Perspectives, CUP, Cambridge, p. 232-246.

SHubert, L. \& Pelletier, J., 1989, Generically speaking, or using Discourse Representation Theory to interpret generics, in G. Chierchia, B. Partee \& R. Turner (eds.), Properties, Types, and Meanings II : Semantic Issues, Kluwer, Dordrecht, p. 193-268.

van der SANDT, R., 1992, Presupposition projection as anaphora resolution, Journal of Semantics, 9, 332-377. 
Svetlana Vogeleer

de Swart, H., 1991, Adverbs of Quantification: A Generalized Quantifier Approach, Groningen University Dissertation, Published in 1993, New York, Garland.

de Swart, H., 1996, (In)definites and genericity, in M. Kanazawa, C. Piñon \& H. de Swart (eds.), Quantifiers, Deduction, and Context, CSLI Publications, Stanford, p. 171-194.

de Swart, H., 2006, Aspectual implications of the semantics of plural indefinites, in S. Vogeleer \& L. Tasmowski (eds.), Non-definiteness and Plurality, Benjamins, Amsterdam, p. 161-189.

de Swart, H. \& MolendiJK, A., 1998, Frequency and tense use in French, Belgian Journal of Linguistics, 12, p. 43-60.

Vogeleer, S. \& TASmowski, L., 2005, Les N, un $N$ et des $N$ en lecture générique, Travaux de linguistique, 50, p. 53-78. 